Bioscientia Medicina: Journal of Biomedicine \&

Translational Research

Journal Homepage: www.bioscmed.com

\title{
Success Rate on Double-J Stenting on Gynecological Cancer Patients: A Meta-Analysis Study
}

\section{Sitti Riri Hafsari ${ }^{*}$, Setya Anton Tusara Wardaya ${ }^{2}$}

${ }^{1}$ Department of Surgery, Faculty of Medicine, Sebelas Maret University, Surakarta, Indonesia

${ }^{2}$ Department of Surgery, Urology Division, Faculty of Medicine, Sebelas Maret University, Surakarta, Indonesia

\section{A R T I C L E I N F O Keywords: \\ Double j stent \\ Gynecological \\ Malignancy \\ Urethral obstruction \\ *Corresponding author: \\ Sitti Riri Hafsari \\ E-mail address: \\ bimariri@gmail.com}

All authors have reviewed and approved the final version of the manuscript.

https://doi.org/10.32539/bsm.v5i4.387

\begin{abstract}
A B S T R A C T
Background. Metastatic cervical cancer in the ureter may lead to obstructive uropathy due to mechanical ureteral blockage. The insertion of a double-J (DJ) stent is a urinary diversion option that can be offered to the patients, but it becomes challenging due to the high rate of failures. This study aims to analyze the success rate on DJ Stent placement on patients with gynecological malignancy. Methods. The study was a systematic review using medical journals collected from Google Scholar, BMJ and PubMed. The journals were searched from 2015 to 2020. The database search through out 2015-2020 using "Double J Stent', "Gynecological", "Malignancy", “Kidney failure", "Hydronephrosis" and "Urethral Obstruction". A literature search was performed and evaluated based on established criteria. The quality assessment of included studies done according to the PRISMA. Meta analysis were done using the fixed effect model or random effect model. Results. The identified article on each med were collected using Mendeley Desktop software. There were 101 scientific publications from PubMed, 232 from Scopus, 1 from Cochrane and 60 from reseachgate results found using the combined keywords. The total of 7 publications matched all the inclusion criteria in this study. Conclusions. Patient's general well-being could be measured by the staging of the malignancies and kidney function laboratory test. Another factor should be thinks through was the side effects of the chemotherapy or radiotherapy, as well as the compliance of the patient during stent maintenance.
\end{abstract}

\section{Introduction}

The prevalence of cervical cancer in Indonesia were about 4,3 per 1000 citizens. According to the International Agency for Research on Cancer, more than $80 \%$ of cancer in the world caused about 273.000 death per year. In Indonesia, during 2014 the number of cervical cancer case were fluctuating, with the case fatality rate of $13,6 \% .1$ Hydronephrosis or hydrouereteronephrosis are the two of the most common complications from cervical cancer. According to the International Federation of Gynecology and Obstetric, the patients with advanced staged of cervical cancer usually have the complication related to pelvic wall, urethra and the disturbance of the kidney functions. ${ }^{2}$ This could cause a vicious cycle' when renal failure and uraemia causes further worsening of gynecological malignancy by increasing neuropathy, sensory changes and obtundation, increasing musculoskeletal cramping, seizures, retention of fluid and electrolyte balance due to vomiting. 3

The malfunctioning of the kidney related to the poor prognositic in cervical cancer. The mean prognositic is approximately less than 12 months. The shorter life expectancy, the more urologists are 
discouraged to perform any major invasive surgery in order to reconstruct the affected urinary tract. Thus, the placement of urethral stent is one of the most common options for the treatment. ${ }^{4}$ The exception of the stent placement is when stenting is not feasible. In that case, percutaneous nephrostomy is the other treatment option. The placement of Double J stent (DJ stent) is more acceptable for the patients and could last for years. ${ }^{5}$ Stent placement was a common temporary relieve for urethral obstruction. The purpose was to aid the draining of the upper urinary tract straight to bladder. The procedure preferred for the placement was usually endoscopic procedure, due to the last invasive procedure. Double $\mathrm{J}$ stent provides self-retaining capability due to its shape. 6

However, the succession of the DJ stent placement was still improving due to the high rate of failures during installation. Several factors such as anesthesia, the equipment preparation and the possibility of time extension were some of the factors influencing the success rate of DJ Stent placement. ${ }^{5}$ Malignancy that cause obstruction has the higher risk of stent placement failure. ${ }^{6}$ Thus, we conduct a meta-analysis study to analyze the success rate on DJ Stent placement on patients with gynecological malignancy. This study included all interventional and observational studies that presented original data of using double $\mathrm{J}$ Stenting on Gynecological Cancer Patients.

\section{Methods}

The Preferred Reporting Items for Meta-Analyses (PRISMA) guidelines for systematic reviews were used.

\section{Search strategy and study selection}

The literature search was developed and conducted by an experienced librarian. The study was a systematic review using medical journals collected from Google Scholar, BMJ and PubMed. We included all interventional and observational studies that presented original data of using double $J$ Stenting on Gynecological Cancer Patients. We included studies presenting data from 2015 to 2020. The search was done by using the following keywords: "Double J Stent', "Gynecological Malignancy", and "Urethral Obstruction".

\section{Data extraction and analysis}

The inclusion criteria were: clinical trial, case report, research survey and meta-analysis that discussed the indication of DJ Stent on Gynecological malignancies, the success rate and the factors contributing to its success and failure. Only full text scientific publications were collected. Single-case studies or case series were excluded, as were studies that were presented only as abstracts, posters, or Letters to the Editor. Articles published in a language other than English were translated and included.

Studies that only enrolled patients with a primary diagnosis of gynecological cancer. That limited the study population to a specific cause, symptom, and/or comorbidity of gynecological cancer as these studies were outside the scope of our study objectives. Other exclusion criteria included studies that were limited to acute or intradialytic pain

The quality assessment of included studies done according to the PRISMA (Preferred Reporting Items for Systematic Reviews and Meta-analysis). We record identified through database searching as 394 articles from PubMed (101 item), Scopus (232 item), Cochrane (1), and Researchgate (60). And we identified from additional records through other sources as 4 articles.

Meta analysis were done using the fixed effect model or random effect model. Fixed effect model assumed that variability among studies based on the probability factor. If the research was carried out indefinitely, the same results will be obtained. The random effects model an addition to intra study variability, inter-study variability is also taken into account. Therefore, a wider confidence interval will be obtained compared to the fixed effects model. The risk of bias and methodological quality of the included studies was explored using the Newcastle-Ottawa Scale (NOS). 




Figure 1. The PRISMA chart of data collection 
Table 1. Characteristics of included studies

\begin{tabular}{|c|c|c|c|c|c|}
\hline Study & Year & Origin & Study Type & Subjects & Result \\
\hline Goldfarb et al & 2017 & Minneapolois, USA & Retrospective & 202 & $\begin{array}{l}\text { The burden of disease is higher } \\
\text { than expected. The } \\
\text { complications were higher on } \\
\text { patients who underwent } \\
\text { radiotherapy }\end{array}$ \\
\hline Duaras \& Oka & 2020 & $\begin{array}{l}\text { Denpasar, } \\
\text { Indonesia }\end{array}$ & Retrospective & 100 & $\begin{array}{l}\text { The successful rate of stent } \\
\text { insertion was } 57 \% \text {. Staging of } \\
\text { cervical cancer, history of } \\
\text { radiotehrapy, albumin levels and } \\
\text { gradiing of hydronephrosis were } \\
\text { the statistically significant } \\
\text { predicting factors. }\end{array}$ \\
\hline Kurata et al & 2016 & Saga, Japan & Retrospective & 33 & $\begin{array}{l}\text { Stent withdrawal and poor } \\
\text { prognosis were associated with } \\
\text { patients with gynecological } \\
\text { malignancies. Enlargement of } \\
\text { lympmh nodes and ureteral } \\
\text { stenosis were two of the } \\
\text { contributing factors in the } \\
\text { failure of DJ Stent placement. }\end{array}$ \\
\hline Kang et al & 2018 & Liaoning, China & Literature Review & 1989 & $\begin{array}{l}\text { Metallic ureteral stents were } \\
\text { statistically has higher sucessful } \\
\text { rate on stent placement } \\
\text { compared to the others. }\end{array}$ \\
\hline Tognarelli et al & 2019 & Pisa, Itally & Case Report & 1 & $\begin{array}{l}\text { The use of monoclonal } \\
\text { antibodies in gynaecological } \\
\text { neoplasm could prevent ureteral } \\
\text { compression. This should be } \\
\text { considered in the management } \\
\text { of gynecological malignancy. }\end{array}$ \\
\hline Hung et al & 2017 & Taipei, Taiwan & $\begin{array}{l}\text { Retrospective } \\
\text { Studies }\end{array}$ & 94 & $\begin{array}{l}\text { The installation of stent failure } \\
\text { mainly due to hydroneprhosis } \\
\text { upgrade, serum creatinine } \\
\text { elevated over } 150 \% \text { baseline and } \\
\text { ipsilateral percutaneous } \\
\text { nephrostomy. }\end{array}$ \\
\hline Tan et al & 2019 & Shenyang, China & $\begin{array}{l}\text { Retrospective } \\
\text { study }\end{array}$ & 89 & $\begin{array}{l}\text { The increasing risk of failure in } \\
\text { the installation of urethral stent } \\
\text { are mainly due to the higher } \\
\text { degree of hydronephrosis and } \\
\text { the length of obstruction }\end{array}$ \\
\hline
\end{tabular}




\section{Result}

\section{Study selection}

From all articles, we identified records after duplicates removed are 325 articles. Then we screened and we identified exclude criteria, so we get 243 articles. From full-text articles assessed for eligibility we get 82 articles and after we identified exclude criteria, we get 62 articles. Based on include studies in qualitative synthesis, we identified 20 articles and last, we identified quantitative synthesis (meta-analysis) about 7 articles.

\section{Meta analysis results}

The database search throughout 2015-2020 using “double j stent', "gynecological”, “malignancy”, "kidney failure", "hydronephrosis" and "urethral obstruction". The identified article on each med were collected using Mendeley Desktop software. Information sources included electronic databases, reference lists of relevant literature, and websites of relevant networks, organizations, and societies. The electronic databases searched included 101 scientific publications from PubMed, 232 from Scopus, 1 from Cochrane and 60 from ResearchGate results found using the combined keywords. The total of 7 publications matched all the inclusion criteria in this study. Five publications were a retrospective study using secondary data dan two case reports.

The research done by Goldfarb et al showed that from the 202 subjects underwent the stent placement surgery, 85 (42\%) underwent one stent placement procedure (no re-treatments), 55 (27\%) were treated twice, and the remaining $62(31 \%)$ were treated 3 or more times, 51 (25\%) had the initial stent procedure in the 12 months prior to cancer treatment, 91 (45\%) in the 12 months after treatment (including on the actual day of treatment), and 60 (30\%) had the initial stent procedure more than 12 months after treatment. The most common initial procedure was cystoscopy with stent placement (57\%, CPT 52332) followed by nephroureteral stent placement via percutaneous approach (20\%, CPT 50393). Similar frequencies were observed for subsequent procedures as well. Stent removal without simultaneous replacement was performed in 80 patients, <11 of whom subsequently had nephrostomy tube placed. The complications reported were lower urinary tract symptoms, hematuria, incontinence, urinary retention, renal colic, urinary stones and urinary tract infections. However, the adverse effect aren't significantly different between the cancer group and non cancer group. ${ }^{7}$

The different results were shown by Kurata et al. From the 45 urethral stents placement surgery on 33 patients, tumor was the most common cause of obstruction, followed by lymph swelling. The success rate of ureteral stent placement was $72.5 \%-79 \%$. The factors reported as the predictors of failure were : baseline serum creatinine, no treatment after retrograde ureteral stent placement, gross tumor invasion noted at cystoscopy and the degree of hydronephrosis(8) Hung et al had published a study on the effectiveness and durability of ureteral tumor stent, the National Taiwan University Hospital experience. This too has similar result where the degree of hydronephrosis and the serum creatinine highly correlates with the succession of the DJ stent installation on tumor-related uretreal obstruction. Gynecological malignancy is the main cause of malignancy that causes urethral obstruction (9). Tan et al on their studies on ureteral stent placement and percutaneous nephrostomy in the management of hydronephrosis secondary to cervical cancer showed slightly different results. The statistic calculation showed the degree of hydronephrosis and the length of urethral obstruction correlated with the degree of succession of DJ Stent installation on patients with gynecological malignancies. ${ }^{4}$

On the closest similar circumstances, Santosa et al had published a study on predictor of successful DJ stent insertion in advanced cervical cancer. The retrospective study was conduct throughout 2017 to 2020 in Sanglah General Hospital. In his study, the degree of hydronephrosis, cancer size, further metastatic to the bladder, higher BUN level, Creatinine level, albumin level and the history of radiotherapy are the factors that statistically could be concluded as the succession factors for DJ stent installation. 5

Kang et al mainly 
focus on the material of the stents concerning the successful rate of the installation. On a meta-analysis study on the advanced stage of cervical cancer, they found out that metallic stent has higher succession rate on the installation compared to the others. It has lower migration rate and no complication reported after six months. However, on patients with intraluminal endometriosis, this should be avoided due to the proliferative endometrium may obstruct the external drainage of metal stents and decrease its patency. 10

Tognarelli et al on the other hand, focused on the angiogenesis inhibitor monoclonal antibody Bevacizumab as the standard treatment for numerous neoplasms. The addition of this agent on radio and chemotherapy improved the survival rate. However, it increases the risk of urinary fistula. In this case report, 40 years old woman was reported to have a migration of double $\mathrm{J}$ ureteral stent to the rectal lumen through tumor necrosis after treatment with Bevacizumab. The same case had been reported earlier, a migration of double $\mathrm{J}$ ureteral stent due to radiotherapy. The use of Bevacizumab had increased in treating gynecology malignancy. On the other hand, the installation of DJ stand use in gynecological malignancy assessment had been increasing. Fistulization between the lower urinary tract and the rectum in the present case was, in our opinion, related to shrinkage and necrosis of the pelvic recurrence caused by angiogenesis inhibitors, which also increased wall fragility of the pelvic organs. In order to mitigate the risk of fistulization the Urologists should try to monitor and treat concomitant urinary tract infections, use hydrophilic stents of low gauge and avoid traumatic manoeuvres during stent substitution. Advances in stent materials, such as antibacterial coating, are likely to reduce the risk of fistulization. 11

\section{Discussion}

The hydronephrosis in gynecologic disorders can be caused by the direct invasion tumor of the ureter, impigment by the mass, metastatic or the enlarged lymph nodes. If the obstruction wasn't treated, there is a high risk of renal failure. Thus, urine diversion is needed to reconstruct the obstruction of urethra. 12 Acute and chronic ureteral obstruction leading to renal failure is common in late-stage gynaecological malignancies. While these can mechanically be relieved with ureteral stents or percutaneous nephrostomies, the benefit of relief and improvement of renal function needs to be considered in the context of their disease progress and overall comfort. Chronic renal failure and uraemia add symptoms that can increase the burden of symptoms to be palliated, for example, increased neuropathy, sensory changes and obtundation, increased musculoskeletal cramping and even seizures, fluid retention, and nausea with abnormal electrolytes. The benefit and risks of an intervention have to be weighed against the anticipated survival of individual patients. If it is a few weeks, there is little benefit from relief of the obstruction during that small window, and potential harm with enhanced pain sensation. Furthermore, dialysis is rarely warranted even for other acute causes of renal failure without obstruction. The only exception would be an acute, reversible event in a patient for whom a single episode of dialysis might treat the underlying renal injury and who would benefit symptomatically from that relief. 3

Double J stent - a device that aids draining the upper tracts directly into bladder - is usually the treatment of choice on the uretheral obstruction. As other stents, this is only a temporary relief of urethral obstruction. Even so DJ Stent has longer duration compared to other stents. Stent-related problems are extremely common. More than $85-90 \%$ of all patients who have an indwelling ureteral stent will report some complication after placement. Complications include irritative voiding symptoms (frequency, urgency, nocturia, and dysuria), flank pain, suprapubic pain, and hematuria. Stent failure can be a major complication of an indwelling ureteral stent. Stent failure can occur in the absence of stent encrustation and in patients with intrinsic or extrinsic obstruction. While most cases of intrinsic obstruction can be successfully managed with stent replacement or definitive repair of the underlying problem, extrinsic or malignant obstruction is more commonly associated with stent failure. ${ }^{6}$ Kouba and colleagues stated that the failure rate of DJ stent in cases of extrinsic ureteric thrombosis due to malignancy is quite high, especially 
if accompanied by persistent hydronephrosis and low back pain, ranging from $16 \%-58 \%$. Failure of DJ stents in ureteric obstruction due to malignancy is also quite high in ureteral distal distal obstruction when compared to proximal or renal pelvic level obstruction.12 5 out of 7 publications analyzed in this study showed similarity regarding the factors of stent failure. Higher creatinine level highly correlated with the succession rate of DJ stent installation. Increasing creatinine and urea levels are related to the degree of ureteral obstruction due to tumor infiltration, and bilateral ureteral obstruction resulted in decreased blood flow and glomerular filtration rate of both kidneys. This mechanism caused an increase in BUN and creatinine levels while decreasing the $24 \mathrm{~h}$ of urine production. Besides obstruction, there were other factors that caused the increased levels of BUN and creatinine in patients with advanced cervical cancer, which include: Dehydration, sepsis, history of diabetes or renal hypertension, and kidney diseases. 5 Hydronephrosis negatively affects the overal survival of cervical cancer patients. Tan et al study showed that patients with mild hydronephrosis and ureteral obstruction $3 \mathrm{~cm}$ in length had a high success rate of ureteral stent placement. The first choice for these patients can be cystoscopic ureteral stent placement. 4 Meanwhile, more severe hydronephrosis and longer length of ureteral obstruction indicate greater severity of obstruction, and ureteral stent placement becomes correspondingly more difficut. Santosa et al conduct a multivariate analysis and it was shown that hydronephrosis grade was a strong predictor of the success of DJ stent insertion. ${ }^{5}$ However, the exact pathomechanism on how hydronephrosis worsened the overal survival of gynecologic cancer patients who underwent the DJ stent installment, still need further studies.

Another factor that should be considered for the succession rate of DJ stent placement were the treatment of choice side effects and the stent material. Tognarelli had reported an endorectal displacement of right ureteral stent following radiochemotherapy and Bevacizumab. It should be kept in mind on a physician during choosing the treatment that the placement of stent rate is high in case of gynecological malignancies. Also, the rate of patient compliance regarding stent maintenance should be considered.

\section{Conclussion}

A meta-analysis study regarding the succession of DJ stent placement on patients with gynecological malignancies showed that patient's general well-being should be highly considered both in decision what assessment should be taken to treat urethral obstruction and the succession rate. Patient's general well-being could be measured by the staging of the malignancies and kidney function laboratory test. Another factor should be thinks through was the side effects of the chemotherapy or radiotherapy, as well as the compliance of the patient during stent maintenance.

\section{Funding}

There is no sources of funding for this meta analysis study.

\section{References}

1. Erniaty W, Woro O, Handayani K. Faktor Yang Mempengaruhi Keterlambatan Diagnosa Ca Serviks Penderita Masa Kuratif di Semarang. Public Heal Perspect J. 2018;2(2):111-6.

2. Zambon JP, Koslov DS, Mihai B, Badlani GH. Bladder and Ureteral Dysfunction Leading to Hydronephrosis and Hydroureteronephrosis in Adults. Urology [Internet]. 2018;117:1-8. Available from: https://doi.org/10.1016/j.urology.2017.11.0 49

3. Mapara RR, Cochrane RM. Obstetrics and gynaecology. Introd to Surg Students. 2017;297-309.

4. Tan S, Tao Z, Bian X, Zhao Y, Wang N, Chen X, et al. Ureteral stent placement and percutaneous nephrostomy in the management of hydronephrosis secondary to cervical cancer. Eur J Obstet Gynecol Reprod Biol [Internet]. 2019;241:99-103. Available from: 
https://doi.org/10.1016/j.ejogrb.2019.08.02

0

5. Santosa KB, Prawira MM, Pramana IBP, Tirtayasa PMW, Yudiana W, Duarsa GWK, et al. Predictor of Successful DJ Stent Insertion in Advanced Cervical Cancer. Open Access Maced J Med Sci. 2020;8(B):882-6.

6. Newman DK, Rovner ES, Wein AJ. Clinical application of urologic catheters, devices and products. Clinical Application of Urologic Catheters, Devices and Products. 2017. 1-242 p.

7. Goldfarb RA, Fan Y, Jarosek S, Elliott SP. The burden of chronic ureteral stenting in cervical cancer survivors. Int Braz $J$ Urol. 2017;43(1):104-11.

8. Kurata S, Tobu S, Udo K, Noguchi M. Outcomes of Ureteral Stent Placement for Hydronephrosis in Patients with Gynecological Malignancies. Curr Urol. 2017;10(3):126-31.

9. Hung SC, Chiang IN, Huang CY, Pu YS. The effectiveness and durability of ureteral tumor stent, the National Taiwan University Hospital experience. Urol Sci [Internet]. 2016;2-5. Available from: http://dx.doi.org/10.1016/j.urols.2017.05.00 2

10. Kang Q, Jiang F, Yu Y, Yang B. Application of metallic ureteral stents in gynecological malignancies: a literature review. Minim Invasive Ther Allied Technol [Internet].
2020;29(1):1-9. Available from: https: / / doi.org/ 10.1080/13645706.2019.157 2626

11. Tognarelli A, Faggioni L, Manassero F, Gadducci A, Selli C. A case report of endorectal displacement of a right ureteral stent following radiochemotherapy and Bevacizumab. BMC Urol. 2019;19(1):1-4.

12. Soetojo S, Nurdin AT. Charateristics of Gynecological Abnormalities and Types of Urine Diversion at $\operatorname{Dr}$ Soetomo Hospital, Surabaya, Indonesia, in Three-Year Period. Folia Medica Indones. 2019; 55(2): 134. 